\title{
IDENTIFICATION OF PIEZOELECTRIC ULTRASONIC TRANSDUCERS FOR MACHINING PROCESSES
}

\author{
Tarek Tutunji ${ }^{1}$, Ashraf Saleem ${ }^{2}$, Mohammad Salah $^{3}$, and Naseer Ahmad ${ }^{2}$ \\ ${ }^{1}$ Department of Mechatronics Engineering, Philadelphia University, Amman, Jordan \\ ${ }^{2}$ Department of Mechanical Engineering, Taibah University, Al-Madinah Al-Monawara, Saudi Arabia \\ ${ }^{3}$ Department of Mechatronics Engineering, Hashemite University, Zarqa, Jordan
}

\begin{abstract}
Smart or active materials expand or deform as a response to external pressure, temperature, or electromagnetic field. The most commonly used active material in actuators is piezoelectric as it relates the electric field to mechanical compression and elongation. However, mathematical modeling of such actuators is tedious and complicated because of the nonlinear behavior. Therefore, there is a need to employ appropriate identification algorithms in order to capture the modeling process of such actuators. In this paper, ultrasonic transducer that is used in ultrasonically assisted machining is adopted as a case study. A component-based model for the piezoelectric is derived and the dynamic response is analyzed. Then, two identification techniques, ARX and ANN, are used to obtain models that mimic the system's dynamic response with minimal error.
\end{abstract}

\section{INTRODUCTION}

The direct piezoelectric effect is the ability of certain materials to generate electric charge in proportion to externally applied force. The inverse piezoelectric effect is their ability to expand under an electric field. Due to these phenomena, piezoelectric materials can be used as both actuators and sensors. Applications include micromanipulation such as suppression of oscillations, micro-robot, micro-pump, and micro-gripper [1].

Piezomotors usage as actuators is based on the conversion of high frequency mechanical oscillations into continuous motion. Advantages of piezomotors are large torque, high resolution, excellent controllability, small time constant, compactness, high efficiency, silent operation, and no electromagnetic induction.

Researchers have been very active in the area of identification and control of piezoelectric material. Ru et al. [2] have derived a mathematical model to describe the hysteresis effect and tested an adaptive control approach for micropositioning experiment. Juhasz et al. [3] described a method for parameter identification of embedded piezoelectric actuators for the use in a real-time FPGA system. In both of these papers, the applied voltage had a frequency range in hertz. Other researchers investigated the identification of the piezoelectric structure properties [4] and developed models for damage identification [5].

Another interesting application is the Ultrasonic Assisted Tuning (UAT) which is a machining technique that is being used to machine hard-to-cut alloys. In this technique, a high frequency vibration (about $20 \mathrm{kHz}$ ) is superimposed on the movement of the cutting tool that results in a reduction of the required cutting force by $60 \%$ [6].

In this work, component-based model is developed for piezoelectric ultrasonic transducer. This transducer converts electric energy into ultrasonic vibration waves by applying external voltage on the piezoelectric stack. The vibration waves travel through the transducer material in the form of deformation and stress waves. The stress wave is then concentrated at the cutting tip to intensify the effort. As mentioned earlier, modeling of such system is complicated and therefore system identification techniques are used in this work in order to predict an appropriate model for the system based on recorded input-output data. The rest of the paper is organized as following: Section 2 presents the component-based model of the system. Section 3 provides two different identification methods. Results are discussed in Section 4 and Section 5 concludes the paper.

\section{COMPONENT BASED-MODELING}

Component-based modeling is an approach where the system is decomposed into its basic components which can be developed and tested individually. Therefore, a component is defined as an entity or subsystem which is designed to perform certain functions and tasks and can be used as a building block in the design or modeling of a larger system. In the context of this study, the ultrasonic transducer is divided into two basic components as shown in Figure 1:

1- PZT component: this component represents the piezoelectric rings which are considered the driving force for the actuator. This component has three inputs and two outputs as depicted in Figure 1.

2- Mass-Spring-Damper (MSD) component: this component represents a mass that is connected with two springs and two dampers as shown in Figure 1. This component has four inputs and two outputs. For ultrasonic transducer two MSD components are required to model the concentrator.
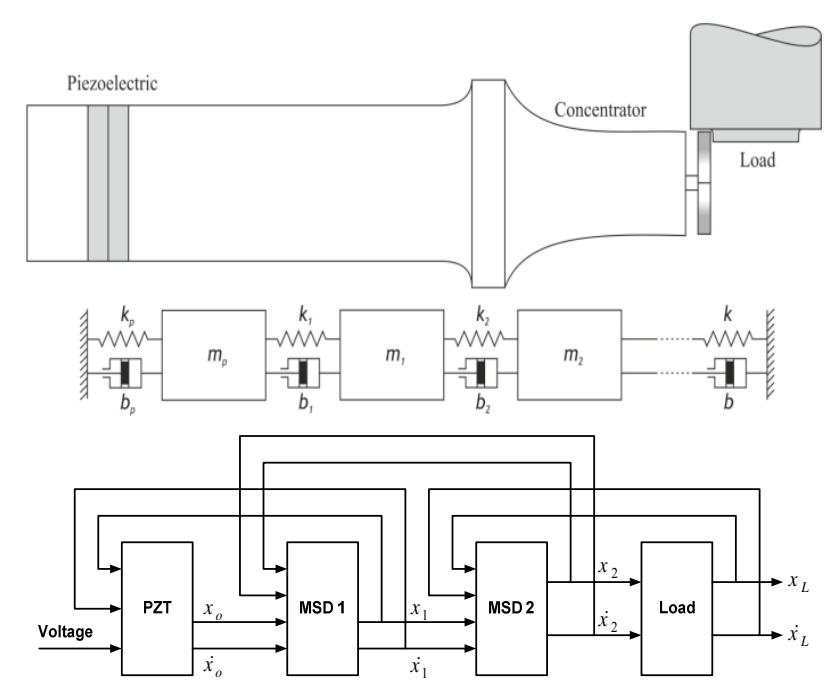

Figure 1. Proposed component-based model for piezoelectric ultrasonic transducers 
The mathematical expression for the PZT can be presented as [7]

$$
m_{p} \ddot{X}_{o}=\left(\frac{T_{e m}}{C c}\right) q-\left(\frac{T_{e m}^{2}}{C c}\right) x_{o}-\bar{F}
$$

and

$$
\bar{F}=k_{p} x_{o}+b_{p} \dot{x}_{o}+k_{1}\left(x_{o}-x_{1}\right)+b_{1}\left(\dot{x}_{o}-\dot{x}_{1}\right)
$$

where $x_{o}(t), \dot{x}_{o}(t)$, and $\ddot{x}_{o}(t)$ denote the displacement, speed, and acceleration of the PZT effective tip, respectively, $x_{1}(t)$, $\dot{x}_{1}(t), x_{2}(t)$, and $\dot{x}_{2}(t)$ are the displacement and speed of the MSD 1 and MSD 2, respectively, $q(t)$ is the charge induced within the piezoelectric, $m_{p}$ is the mass of the piezoelectric material, $T_{e m}$ is the elongation constant, $C_{c}$ is the internal capacitance, $k_{p}, b_{p}, k_{1}, b_{1}$, are the spring constants and damping coefficients of the PZT and MSD 1, respectively, and $\bar{F}$ is the perpendicular force acting on the piezoelectric actuator.

The inherent nonlinear hysteresis (i.e., $\left.q=H\left(V_{h}\right)\right)$ can be modeled as provide by Coleman-Hodgdon model [8]

$$
H=F+d
$$

where $V_{h}=V-V_{c}$. The signal $V_{c}(t)$ is the voltage across the internal capacitance of the piezoelectric and $V(t)$ is the control input (voltage applied on the piezoelectric component). The function $f\left(V_{h}\right)$ is a subsequently defined signal and the function $d\left(V_{h}\right)$ is defined as

$$
d(\cdot)=\left\{\begin{array}{l}
{\left[q_{o}-f\left(V_{h_{o}}\right)\right] e^{-\delta V_{h}-V_{h_{o}} \mid}} \\
+e^{-\delta V_{h}} \int_{V_{h_{O}}}^{V_{h}}\left(g(\tau)-f^{\prime}(\tau)\right) e^{\delta \tau} d \tau, \text { when } \dot{V_{h}(t)>0} \\
{\left[q_{o}-f\left(V_{h_{o}}\right)\right] e^{\delta V_{h}-V_{h_{o}} \mid}} \\
-e^{\delta V_{h}} \int_{V_{h_{o}}}^{V_{h}}\left(g(\tau)-f^{\prime}(\tau)\right) e^{-\delta \tau} d \tau, \text { when } \dot{V_{h}(t)<0}
\end{array}\right.
$$

where $q_{o}$ is the induced charge at $t=t_{o}, V_{h_{o}}$ is the input voltage at $t=t_{o}, \delta$ is a positive constant, $f^{\prime}(\tau)=\frac{\partial f\left(V_{h}\right)}{V_{h}}$ and $g(\cdot)$ is a subsequently designed signal. To validate the expressions in equation (4), the functions $d\left(V_{h}\right), f\left(V_{h}\right)$ and $g\left(V_{h}\right)$ have to satisfy certain properties that can be found in [7, 8]. As for the MSD 1 and MSD2 models, they can be expressed as

$$
\begin{gathered}
m_{1} \ddot{x}_{1}=-b_{1}\left(\dot{x}_{1}-\dot{x}_{0}\right)-k_{1}\left(x_{1}-x_{0}\right) \\
+b_{2}\left(\dot{x}_{2}-\dot{x}_{1}\right)+k_{2}\left(x_{2}-x_{1}\right) \\
m_{2} \ddot{x}_{2}=-b_{2}\left(\dot{x}_{2}-\dot{x}_{1}\right)-k_{2}\left(x_{2}-x_{1}\right)
\end{gathered}
$$

where $m_{1}$ and $m_{2}$ are the mass of the concentrator's parts; $b_{1}$ and $b_{2}$ are its damping coefficients; $k_{1}$ and $k_{2}$ are its stiffness coefficients. These parameters are estimated by creating a full model of the system using Finite Element Method. This was achieved by finding the resonant frequencies of the system and then used to calculate the stiffness and damping coefficients.

\section{SYSTEM IDENTIFICATION}

System identification is the field of approximating mathematical models by measuring experimental input and output data of the system under test. The identification models can be parametric or nonparametric, linear or nonlinear. In this paper, two parametric methods are used: (1) linear method that uses Auto Regressive with eXogenous Input (ARX), and (2) nonlinear method that uses Artificial Neural Networks (ANN).

The general block diagram for identifying systems is provided in Figure 2 where the objective is to find the appropriate model to minimize the error between the system's output $y$ and the models output $\hat{y}$ [9]. In Figure 2, the input is denoted by $u$ and the noise is denoted by $n$.

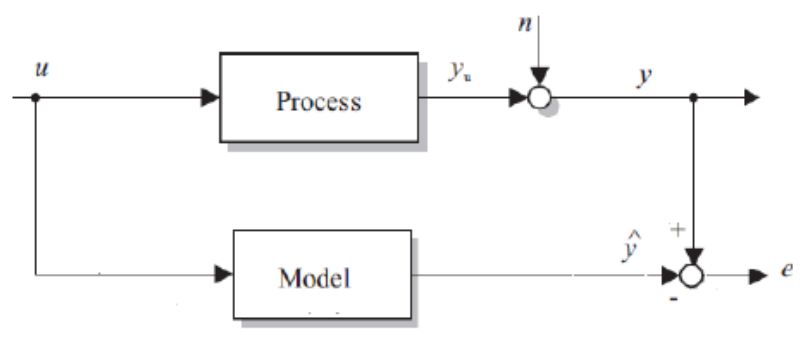

Figure 2. System Identification Block Diagram

One method that is used for system identification is the ARX which combines a deterministic model with a stochastic disturbance. The ARX model are given by

$$
A(q) y(k)=B(q) u(k)+v(k)
$$

where $\mathrm{A}(\mathrm{q})$ and $\mathrm{B}(\mathrm{q})$ are a polynomial functions, $\mathrm{q}$ is the delay, and $\mathrm{u}(\mathrm{k}), \mathrm{y}(\mathrm{k})$, and $\mathrm{v}(\mathrm{k})$ are the input, output, and noise, respectively. The equation can be re-written as

$$
\begin{aligned}
y(k)+a_{1} y(k-1)+\ldots & +a_{n} y(k-n) \\
& =b_{o} u(k)+\ldots+b_{m} u(k-m)+v(k)
\end{aligned}
$$

The ARX block diagram is shown in Figure 3 where the stochastic signal $\mathrm{v}$ is used to model the noise in the real system. This signal is usually modeled as a white noise.

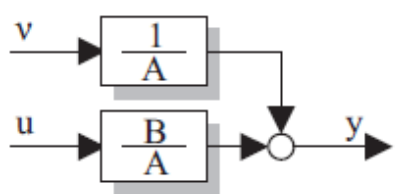

Figure 3. ARX block diagram

By taking the z-transform of equation (7), the transfer function would be

$$
G(z)=\frac{Y(z)}{U(z)}=\frac{b_{o}+b_{1} z^{-1}+\ldots+b_{m} z^{-m}}{1+a_{1} z^{-1}+\ldots+a_{n} z^{-n}}
$$

The effect of the noise is eliminated as the transfer function is used to model the output with respect to the input only. As the noise used is white, it has an average of zero. 
The ANN can also be used for identifying dynamic systems. The advantage of the ANN models is that they can be used for highly-nonlinear systems. A general recurrent multilayer network is shown in Figure 4. This is a multi-input singleoutput configuration. The network accepts the present and past samples of the input $u$ as well as past samples of the output $y$ as input signals and provides one output, y. The network contains activation functions ( $\mathrm{f}$ and $\mathrm{g}$ ) and adapts its output by optimizing its weight matrices ( $\mathrm{W}$ and $\mathrm{V}$ ) to minimize a sum square error between the original and network outputs. The back propagation algorithm can be used for optimizing the weights.

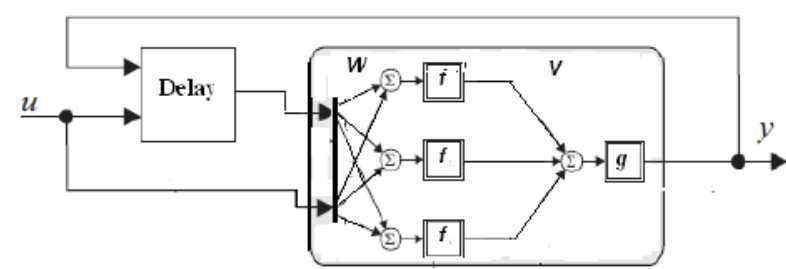

Figure 4. Architecture for recurrent multi-layer neural network

From Figure 4, it can bee seen that the network output, y, is provided by the following equation

$$
y(k)=g\left(\sum_{j}^{n} v_{j} n e t_{j}(k)\right)
$$

where net ${ }_{j}(k)$ is the output from the hidden node $\mathrm{j}$ at pattern $\mathrm{k}$ and is given by

$$
\operatorname{net}_{j}(k)=f\left(\sum_{i} w_{i j} u_{i}(k-i)+\sum_{i} w_{i j} y_{i}(k-i)\right)
$$

Usually, the activation functions used are nonlinear, such as the sigmoid and hyperbolic tangent.

\section{SIMULATION RESULTS}

The component-based model for the ultrasonic transducer was created in Matlab/Simulink. All equations and system parameters used were based on the modeling introduced in Section 2. A sinusoidal input voltage with a frequency of $20.7 \mathrm{kHz}$ was applied to the system. The output displacement of the PZT, MSD 1, and MSD 2 are shown in Figure 5.

Input-output patterns from the component-based model were gathered and used in ARX models to identify the system. A pulse with amplitude of $5 \mathrm{kV}$ was used and the impulse response with 8,000 data points was gathered. The sampling time was $1 \mu \mathrm{sec}$ and therefore the time period was $8 \mu \mathrm{sec}$. For all ARX models, the least squares estimation method was used to find the models' parameters [9].

A simple ARX $(6,2)$ was used to identify the system. This model has six parameters for the A polynomial and two parameters for the B polynomial in equation (7). The results are shown in Figure 6. The ARX results stack almost-perfectly over the component-based model data. In order to have better insight into the results, the residues are shown in Figure 7.
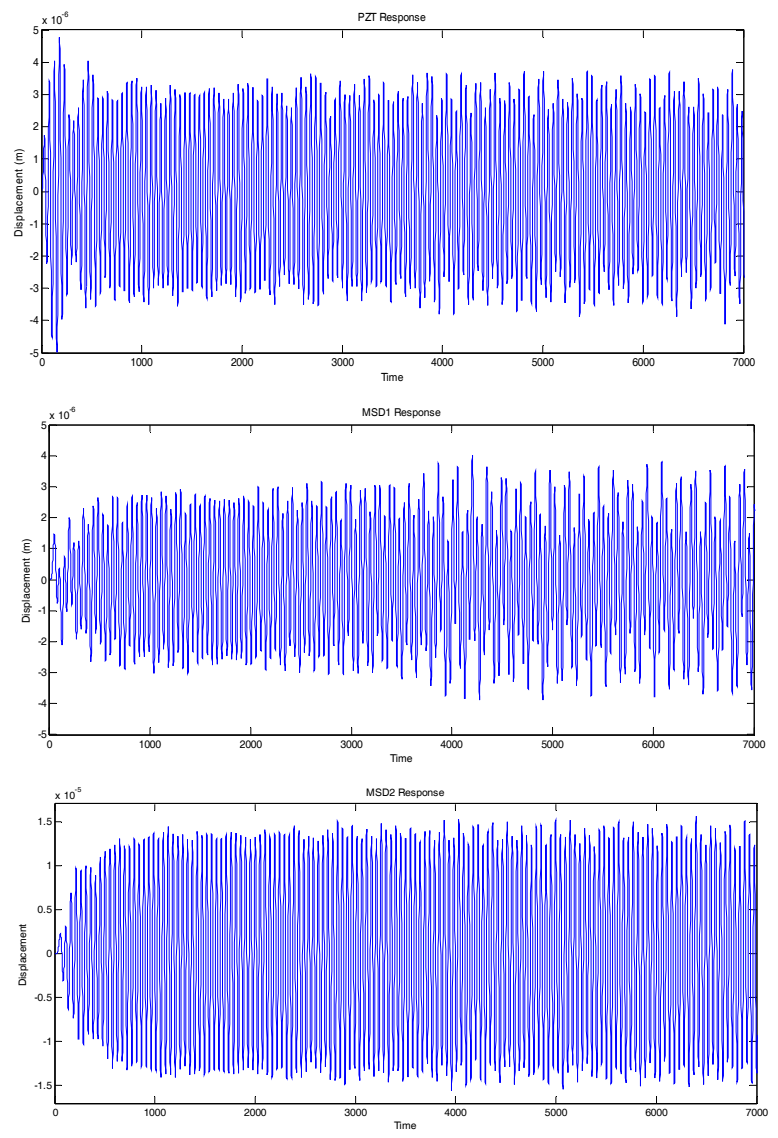

Figure 5. (a) PZT displacement (b) MSD 1 displacement (c) MSD 2 displacement

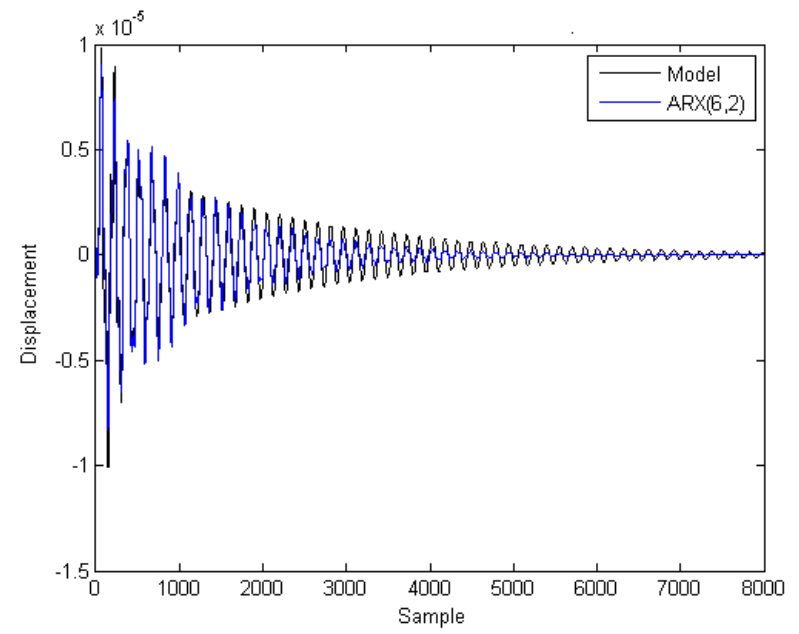

Figure 6. Impulse response comparison: componentbased model vs. ARX $(6,2)$

Once the identified ARX model was realized, it was applied to test data of sinusoidal inputs. The results are shown in Figure 8. Here, the ARX response is given in yellow while the component-based model is given in black. It is clear that the model was not able to follow the test data and that the error is too large. This is due to the fact the piezoelectric model is nonlinear while the ARX model is linear.

In order to compensate for the nonlinearity, the model order was increased: a model of $\operatorname{ARX}(40,10)$ was used (i.e., 40 and 10 parameters for polynomials $\mathrm{A}$ and $\mathrm{B}$ respectively in equation (7)). Figure 9 shows the impulse response data (ARX model 
results are in purple). As predicted, the $\operatorname{ARX}(40,10)$ was able to track the component-based model data.
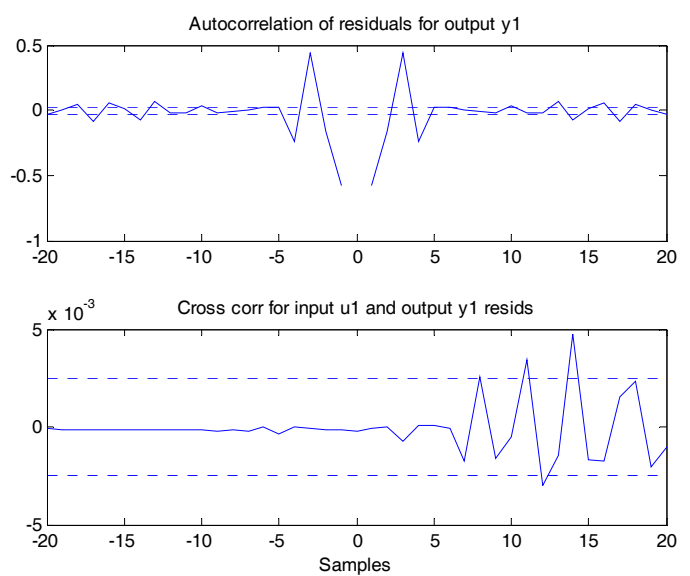

Figure 7. Residual analysis of the ARX $(6,2)$ model

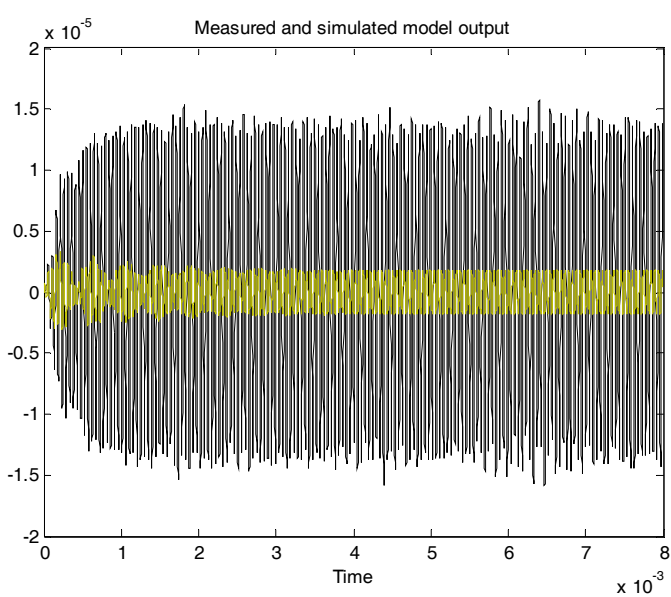

Figure 8. Test Data Comparison: component-based model vs. $A R X(6,2)$

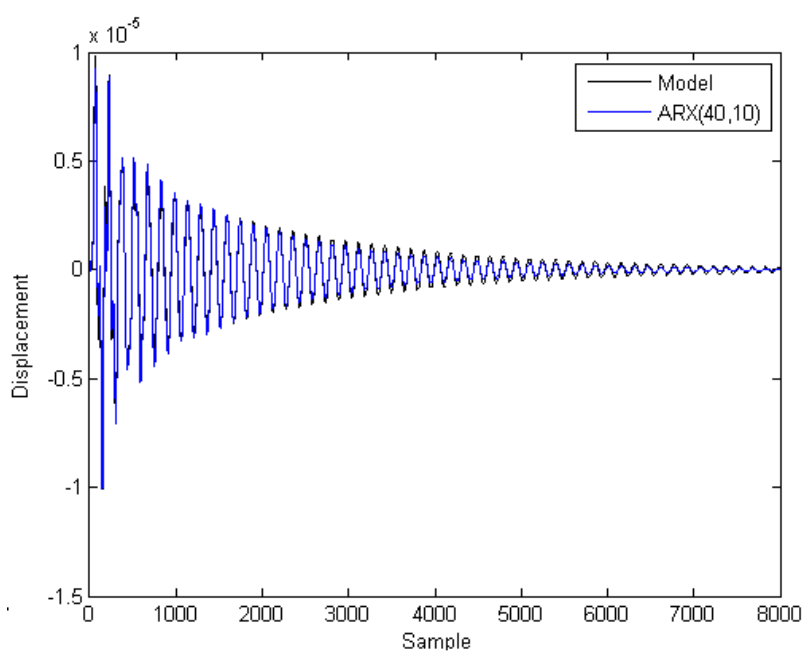

Figure 9. Impulse response comparison: componentbased model vs. ARX $(40,10)$

The ARX $(40,10)$ identified model was then used to predict the sinusoidal test data. The results are shown in Figure 10 for the 8,000 data points and Figure 11 for the first 1,000 points. It is clear that, unlike the lower-order $\operatorname{ARX}(6,2)$, this higher-order identified model was successful in predicting the system's test data behavior.

Next, ANN was used to identify the system. For all ANN runs, the backpropagation algorithm (with LevenbergMarquardt training) was used. Also, the hyperbolic tangent was used as the activation function for all neurons and the meansquare-error was used as the performance function [10].

A recurrent multi-layer network with a single hidden layer (10 hidden nodes) was simulated. The network had five input signals: $\mathrm{u}(\mathrm{k}), \mathrm{u}(\mathrm{k}-1), \mathrm{y}(\mathrm{k}-1), \mathrm{y}(\mathrm{k}-2), \mathrm{y}(\mathrm{k}-3)$; and one output, $\mathrm{y}(\mathrm{k})$. Here, the sinusoidal data was divided into two parts: training data (first 5,000 points) and testing data (last 3,000 points). The training results are shown in Figure 12. By comparing the two figures, it can be seen that the ANN was able to provide similar output to the component-based model. In order to get a better view, only the first 500 data points are shown in Figure 13.

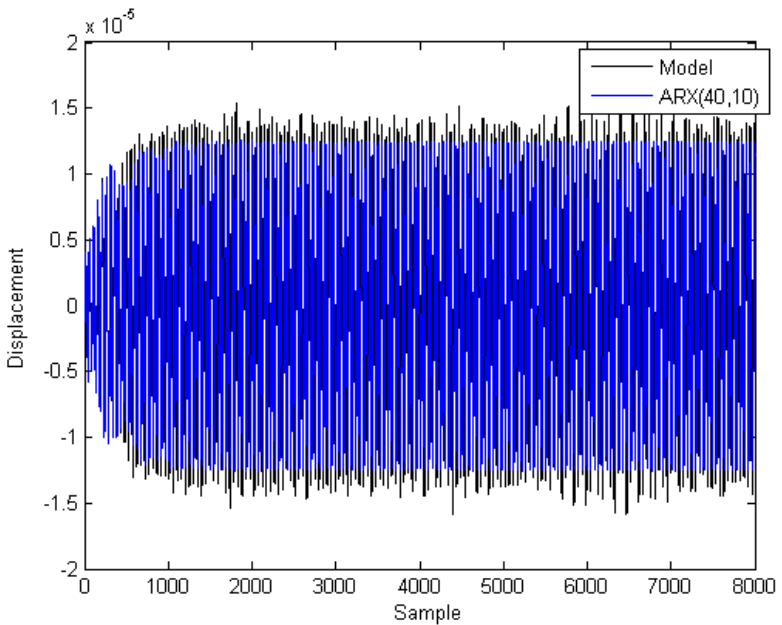

Figure 10. Test data comparison: component-based model vs. ARX $(40,10)$

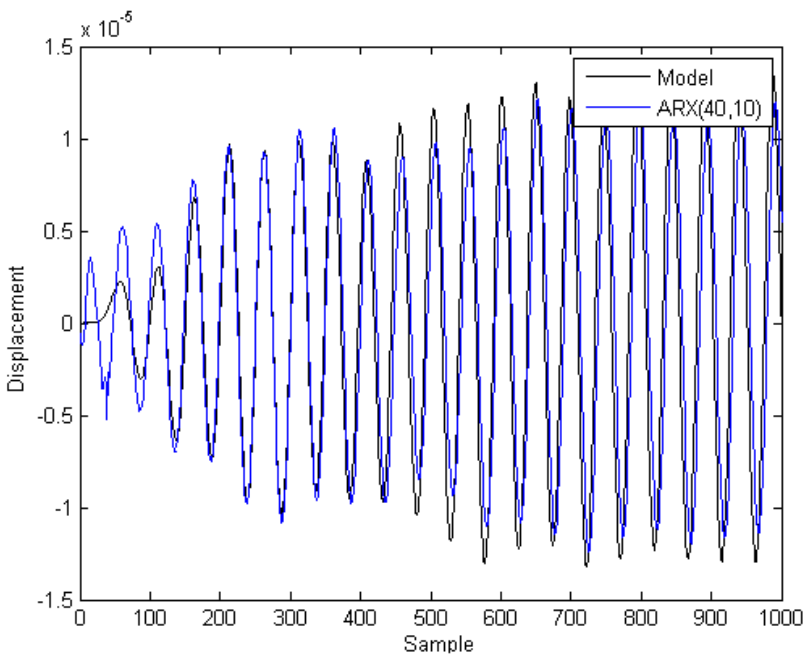

Figure 11. Test Data comparison zoom-in: componentbased model vs. ARX $(40,10)$

Then, the trained network was tested for the last 3,000 data points. In order to display an appropriate view, 500 data points are shown in Figure 14. It is clear that the ANN predicts the testing data with minimal error. 

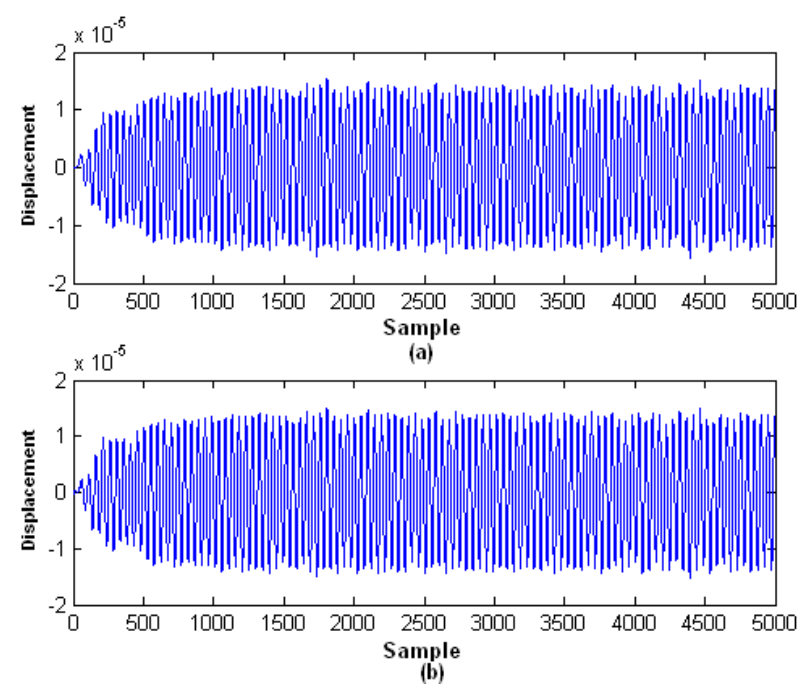

Figure 12. Output results for training data: (a) Componentbased model (b) ANN

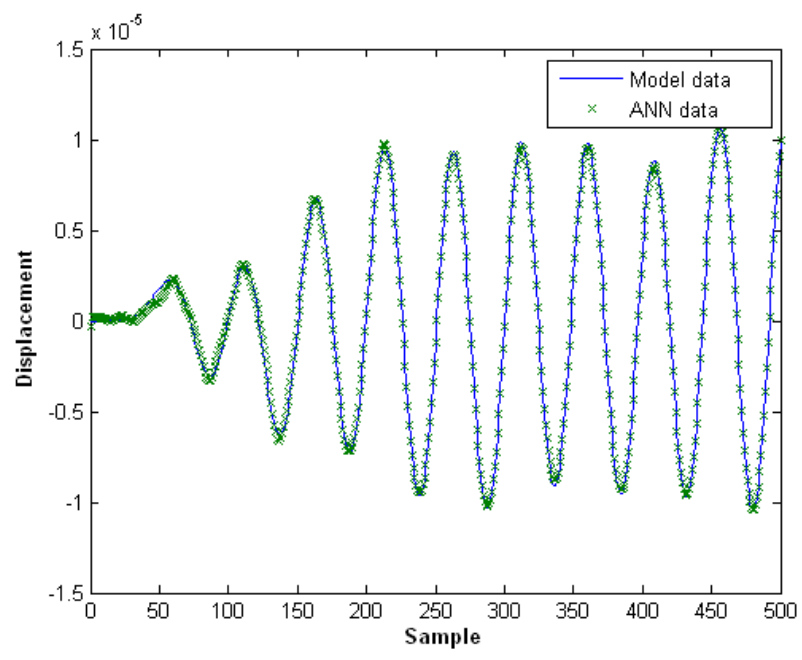

Figure 13. ANN vs. component-based models: results for the training data (zoom-in 500 data points)

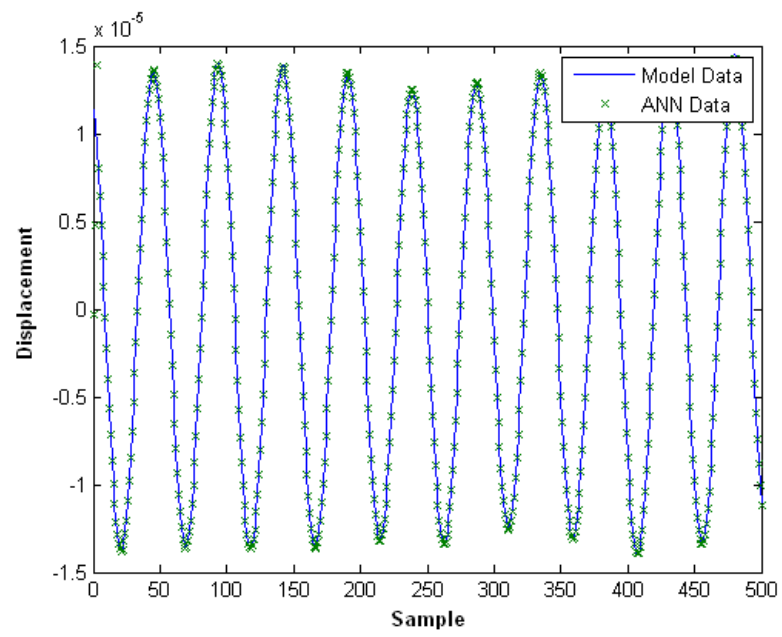

Figure 14. ANN vs. component-based models: results for the testing data (zoom-in 500 data points)

\section{CONCLUSIONS}

This paper presented a component-based model of piezoelectric ultrasonic transducers and used two system identification techniques (ARX and ANN) to identify the system. The results showed that the low-order ARX model identified the training data only while a high-order ARX identified the training and testing data. This is due to the fact that the piezoelectric ultrasonic transducer model is nonlinear while the ARX is a linear model and therefore it requires higher-order models to compensate for the nonlinearities. On the other hand, the ANN, a nonlinear model, identified both the training and testing sinusoidal data. Work is underway to test physical piezoelectric-based systems and collect real-time experimental data in the labs. Identified models can then help in predicting the material's behavior and in designing appropriate controllers.

\section{ACKNOWLEDGMENT}

This research was supported by the Strategic Technologies Program of the National Plan for Science, Technology and Innovation in the Kingdom of Saudi Arabia under the project no. 09-ADV809-05.

\section{REFERENCES}

[1] Ramutis Bansevicius and Rymantas Tadas Tolocka, "Piezoelectric Actuators". Mechatronics Handbook by Bishop. 2002. Section 20.3 Page 575

[2] $\mathrm{Ru}$, Chen, Shao, Rong, and Sun. A hysteresis compensation method of piezoelectric actuator: Model, identification, and control. Control Engineering Practice 17 (2009) 1107 - 1114.

[3] Juhasz, Mass, and Borovac, Parameter identification and hysteresis compensation of embedded piezoelectric stack actuators. Mechatronics 21 (2011) 329-338

[4] Rus, Palma, Perez-Aparicio. Experimental design of dynamic model-based damage identification in piezoelectric ceramics. Mechanical Systems and Signal Processing 26 (2012) 268-293

[5] Montemurro, Nassar, Koutsawa, Belouettar, Vincenti, and Vannucci, Identification of electromechanical properties of piezoelectric structures through evolutionary optimization technique. International Journal of Solids Structures 49 (2012) 1884-1892.

[6] Ahmed, N., et al., Analysis of forces in ultrasonically assisted turning. Journal of Sound and Vibration, 2007. 308(3-5): p. 845-854.

[7] M.H. Salah, M.L. McIntyre, D.M. Dawson J.R.Wagner, and E.Tatlicioglu. Charge feedback-based robust position tracking control for piezoelectric actuators. IET Control Theory and Applications 2012 Vol. 6, Issue 5, pages 1-14

[8] Coleman, B., Hodgdon, M.: 'On a class of constitutive relations for ferromagnetic hysteresis', Arch. Ration. Mech. Anal., 1987, 99, (4), pp. 375-396

[9] Rolf Isermann and Marco Muchhof. Identification of Dynamic Systems: An Introduction with Applications. Springer 2011.

[10] Simon Haykin. Neural Networks and Learning Machines. Prentice Hall 2008. 